\title{
A Mathematical Model of U.S. COVID-19 Deaths, Incidence and Quarantine Adjusted Herd Immunity by Age
}

Vikki Lovvoll ( $\sim$ vikki.lovvoll@gmail.com )

N/A

Research Article

Keywords: COVID-19, herd immunity, prediction model

Posted Date: April 30th, 2021

DOl: https://doi.org/10.21203/rs.3.rs-343570/v1

License: (9) This work is licensed under a Creative Commons Attribution 4.0 International License. Read Full License 


\section{Abstract}

In January 2021, a steep decline in U.S. COVID-19 deaths and case reports were noted, well before herd immunity or vaccinations would have been expected make an impact. This model predicts COVID-19 deaths and infections based on three insights. First, mortality rates differ significantly by age. Second, heterogeneous social mixing by age during quarantine may result in older adults (higher risk) to have more effectively quarantined conferring lower incidence relative to younger adults (lower risk and disproportionate essential frontline work). Third, deaths and Infection Fatality Rates may more reliably predict incidence relative to reported cases by removing uncertainty introduced from asymptomatic disease, testing availability, and false results. Age stratified IFR and deaths through December 5 were used to projected deaths ( $99 \%$ significance, P-value .002). Sensitivities for IFR, immune durability, and vaccinations were also modeled. By end $2020,42 \%$ of the U.S. population had immunity differing significantly by age with $25-44$-year-olds near $80 \%$. A "critical mass" of immunity in these ages segments conferred herd immunity to other age groups. Herd immunity exists in the context of historic social distancing (quarantine). A significant percent of high-risk individuals remains susceptible and may facilitate another wave if social distancing restrictions are lifted prior to vaccination. Vaccine administrations through mid-February contributed a nominal amount to declining deaths.

\section{Background And Purpose}

CDC reported COVID-19 cases and deaths ${ }^{(1)}$ peaked and waned several times throughout 2020. (Figure 1) While geographic hot spots, quarantine and/or seasonal weather patterns could have reasonably contributed to historic patterns, these variables are unlikely to explain the steep decline in January 2021. While some hypothesize that the January decline may be herd immunity, others argue estimates of cumulative incidence ${ }^{(2)}$ are significantly lower than the minimum required for herd immunity based on traditional Susceptible, Exposed, Infected, Recovered (SEIR) models ${ }^{(3)}$. Further, even assuming 95\% vaccine efficacy after two doses and $80 \%$ vaccine efficacy after one dose, the volume of vaccinations to date also cannot reasonably account for this decline. SEIR estimates of herd immunity thresholds have historically been modeled in the context of an unrestricted social structure and/or do not adjust for heterogeneity in social mixing due to disproportionate risk and essential work exposure. Several studies have sought to contextualize the impact of heterogenous social mixing on community disease spread but have not quantified the relationship to incidence and deaths with real world data. ${ }^{(4)}$ (5) This novel mathematical model uses publicly available data to quantitatively describe the relational sensitivity of age stratified risk and social mixing to predict the timing and magnitude of death trends and quarantine adjusted thresholds for herd immunity.

The hypothesis is that given variable risk (Infection Fatality Rates) by age, older adults have taken more extreme social distancing measures and therefore have lower cumulative incidence and immunity relative to younger adults who disproportionally make up frontline essential workers. As a result, younger adults with lower mortality risk have achieved a critical mass of actual exposure/immunity due to high cumulative incidence rates while older adults with much higher risk continue to be at risk. Further, an 
unfortunate side effect of younger adults being frontline essential workers is that they may be, as a cohort, inadvertently acting as "super spreaders". Conversely, after achieving critical mass of exposure/immunity they may be contributing significantly to quarantine adjusted herd immunity.

This analysis could contribute to the growing body of knowledge in COVID-19 disease epidemiology, age and time variable susceptibility, public policy considerations and vaccination strategies which can optimize population health outcomes, particularly in a constrained vaccine supply environment.

\section{Methods}

COVID-19 disease Incidence is difficult to quantify for several reasons including variability over time, variability across geographies, availability of testing, false positive/negative results, and asymptomatic disease. COVID-19 related Deaths are more of a known variable because all states report COVID-19 deaths and year-over-year comparative analysis provide additional confidence in quantifying incremental deaths attributed to COVID-19. Using COVID-19 IFR to estimate COVID-19 incidence removes the additional variability introduced by testing (availability, test rate, false positive/negatives, etc). COVID-19 incidence can be estimated from deaths using the Infection Fatality Ratio (IFR) which is "the number of individuals who die of the disease among all infected individuals (symptomatic and asymptomatic)" (6) such that deaths divided by IFR yields the incident population and, when divided by the total population, yields the incidence. Additionally, total population size multiplied by IFR yields the theoretical maximum number of deaths (assuming a 100\% infection rate). Finally, since deaths are reported each week and by age group it is possible to estimate weekly COVID-19 incidence trends from age specific COVID-19 IFRs.

\section{Results}

Historical Reported Deaths

CDC reported COVID-19 deaths by age from week ending February 1, 2020 through week ending January $2,2021^{(19)}$ show that while $7 \%$ of the population is $75+$ years old, they accounted for $58 \%$ of reported deaths. (Figure 8) Older adults consistently accounted for the most COVID deaths over time. (Figure 9).

\section{COVID-19 Incidence and Immunity}

This analysis suggests that, assuming $100 \%$ of the population were infected with COVID, $96 \%$ of the theoretical deaths would be among individuals 65 years old or over and $81 \%$ among those 75 and older. (Figure 10).

Figure 11 shows that through January 2, 2021, despite older age groups accounted for the greatest number of deaths, modeled total infections were greatest among the 35-44-year age group followed by the 25-34 and 45-54 age groups then 15-24 and 55-64 age groups. Collectively these younger adult segments disproportionally make up the essential frontline workers that had the greatest exposure. 
Figure 12 shows that by the end of 2020 cumulative immunity was greatest among the 35-44-year age group (80\%) and lowest among the oldest (6-12\%) and youngest age segments (2-16\%) making a "bell curve" while Figure 13 shows total (age weighted) cumulative population immunity trends approached $42 \%$ by January 2021 in line with consensus estimates by epidemiology experts.

\section{Projected Deaths, Infections, \& Immunity}

Figure 7 shows that older age groups not only had the most deaths but were increasing at the greatest rate and, because of low cumulative immunity among this segment, trends continue to accelerate through summer 2021 in the absence of interventions (natural, behavioral or vaccination).

At the total population level, an inflection of total infections was predicted to peak in early January then decline precipitously (Figure 15) while unadjusted death trends (Raw Projected Deaths) theoretically continued to accelerate (Figure 14). This is due to younger adults achieving maximum projected deaths (not herd immunity but immunity through $100 \%$ of population exposed) with minimal impact on deaths due to low IFRs in this age group. Conversely, older adults continued to be at risk due to low cumulative incidence/immunity and therefore continued to disproportionally contribute to deaths. However, model fit of Raw Projected Deaths to Reported Deaths was poor in the projection period ( $r$ value -.626). Given the significant decline in projected infections and the actua/ decline in deaths the hypothesis of herd immunity was considered. In support of this hypothesis, reported deaths vs unadjusted raw projected deaths was evaluated by age group. In addition to the high immunity among the 35-44 age group, raw Projected Deaths closely aligned to Reported Deaths in this segment $(r=0.693)$ and are negatively correlated by an increasing amount for older and younger age groups suggesting that the 35-44 age group is "leading the herd".

\section{Quarantine Adjusted Herd Immunity}

After including a variable to adjust for the impact of head immunity from declining infections among the 35-44-year age segment (see Methods for calculations) the resulting Quarantine Adjusted Herd Projected Deaths align very closely to actual reported deaths in total and across all age groups (Figure 16). A Pearson Correlation between Reported Deaths and Projected Deaths in the hold out period (December 6 February 13) was used to evaluate the null hypothesis to determine if herd immunity contributed to the decline in deaths. Herd Immunity Adjusted Deaths demonstrated a .910 rvalue relative to Reported Deaths and supports a rejection of the null hypothesis with $99 \%$ significance ( $p$-Value <.002). Further, Herd Immunity Adjusted Deaths are predictive at each age segment (see appendix).

\section{Vaccination Impact on Projected Deaths}

Actual and projected vaccinations were adjusted for efficacy and age distribution and applied to raw projected death trends to determine the vaccine adjusted deaths to test the hypothesis that vaccinations contributed to the decline in deaths (see Methods for calculations). A Pearson Correlation between Reported Deaths and Vaccine Adjusted Projected Deaths in the hold out period (December 6 - February 13) was used to evaluate the null hypothesis. Vaccine Adjusted Deaths have a negative correlation to 
Reported Deaths ( $r$ value -.596) and thus do not support a rejection of the null hypothesis that vaccines were the driver in the decline of reported cases and deaths.

This analysis shows that the number of vaccine administrations, when adjusting for lag time and first and second dose efficacy, had nominal impact on death trends prior to mid-February, is a poor predictor of death trends and therefore is unlikely to have meaningfully contributed to the recent decline in deaths.

\section{Sensitivity Analysis}

The sensitivity on model predictability of Reported Deaths was evaluated across eight key variables including a low and high estimate for each and three scenarios (Raw Projected deaths, Herd Immunity Adjusted Deaths, Vaccine Adjusted Deaths). Variables tested included:

- two variables related to Infectious Fatality Rate (value and relative improvement over time),

- two variables on naturally acquired immunity (percent infected that achieve immunity and durability of immunity) and

- three variables on vaccines (efficacy after first dose, efficacy after second dose and lag time between vaccine administration and deaths prevented).

- One variable related to herd immunity (\% infected prior to herd immunity)

Weekly data was stratified into four time periods.

- Week ending 2/1/20 thru 9/2/20: pre-model trend period (changes in weather, social distancing, and geographic spread of disease may have higher influence in infection and death trends),

- Week ending 9/12/20 thru 12/5/20: trend period used in all analysis include Reported Death trends and Herd Immunity coefficients,

- Week ending 12/12/20 thru 1/30/21: hold out period where modeled deaths were correlated to actual reported deaths,

- Week ending 2/6/21 thru 4/17/21: future time (actual reported deaths are not yet available).

None of the sensitivities improved predictability of the model. (Table 1 and Charts 18-20) Due to relatively low volume of individuals with immunity in the first six months of the pandemic and low levels of vaccinations to date, vaccine and immunity related sensitivities are underpowered to reasonably be expected to have an impact.

\section{Table 1}




\begin{tabular}{|c|c|c|c|c|}
\hline & & \multicolumn{3}{|c|}{ Herd } \\
\hline & & & Immunity & Vacaine \\
\hline & & Raw & Adjust ed & Adjusted \\
\hline & & Projection & Projection & Projection \\
\hline \multicolumn{2}{|l|}{ Pears on's Correlation $[r]$} & -62.696 & $91.0 \%$ & -59.696 \\
\hline \multicolumn{2}{|l|}{ P-Value (95\%) } & \multicolumn{3}{|c|}{0.002} \\
\hline Sensitivities & Value & \multicolumn{3}{|c|}{ Relative Imp rovement to $r$} \\
\hline LOW IFR & $1.10 \%$ & 0.096 & $-92.1 \%$ & -2.29 \\
\hline Base IFR & 1.4496 & $-1.1 \%$ & $-60.0 \%$ & $-1.8 \%$ \\
\hline High IFR & $1.95 \%$ & -1.396 & $-160.9 \%$ & -1.796 \\
\hline Low IFR Reduction & $50 \%$ & $1.6 \%$ & -72.396 & $1.3 \%$ \\
\hline High IFR Reduction & $0 \%$ & -1.396 & $-160.2 \%$ & -1.796 \\
\hline Low \% Previously Exposed & $80 \%$ & $-1.3 \%$ & $-0.6 \%$ & $-1.3 \%$ \\
\hline Base \% Previously Exposed & $90 \%$ & -0.796 & & $-0.1 \%$ \\
\hline Base Immune Durability & $12 \mathrm{mo}$ & 0.096 & -153.696 & 0.096 \\
\hline Low Dose 1 Vaccine Efficacy & $50 \%$ & & & $-0.9 \%$ \\
\hline High Dose 1 Vaccine Efficacy & 9096 & & & $0.3 \%$ \\
\hline Low Dose 2 vaccine Efficacy & $096(+4096)$ & & & 0.096 \\
\hline High Dose 2 Vaccine Efficacy & $95 \%(+5)$ & & & $0.0 \%$ \\
\hline Low Vaccine Lag Time & 2 wks & & & 2.896 \\
\hline High Vaccine Lag Time & $6 w k s$ & & & $-1.69 \%$ \\
\hline Low Herd Threshold & $65 \%$ & & $-80.1 \%$ & \\
\hline Base Herd Threshold & 7596 & & -28.99 & \\
\hline
\end{tabular}

\section{Discussion}

The significant decline in COVID-19 cases noted in mid-January came in the middle of winter, just after the holidays, when many experts anticipated a surge in cases, and before vaccines would have been expected to make a significant impact. This mathematical model proposes a plausible explanation for the decline in deaths using age stratified infectious fatality rates, $C D C$ reported deaths and a heterogeneous social mixing matric by age to model naturally derived immunity and predict future death trends. This model quantifies the potential impact of quarantine adjusted herd immunity and vaccinations on predicted deaths and suggests that by end of $2020,42 \%$ of the total population had naturally derived immunity to COVID-19 with significant variability by age. Children ages 1-14 and adults $65+$ years old are estimated to have had less than $12 \%$ cumulative immunity by end 2020 while $25-44$-year-olds had an estimated 78$80 \%$ immunity. These trends may reflect higher exposure due to heterogeneity of social mixing across ages because of essential work and more relaxed behaviors among younger adults, who have a relatively lower risk, and lower exposure among older adults who have a significantly higher risk and be more likely to observe a stricter quarantine. An analysis of age stratified reported deaths and IFR yielded levels of naturally derived immunity that achieve "critical mass" (quarantine adjusted herd immunity threshold) among 34-44-year-olds by late 2020. Regression analysis quantifying the relationship between total projected infections in period $t_{x n}$ and age stratified projected infections in period $t_{x n+1}$ remains static preand post- the recent decline in deaths. This demonstrates that the decline in reported deaths among the 34-44-year-old segment--as a result of achieving "critical mass" of naturally acquired immunity-can explain the decline in reported deaths among other age groups by way of quarantine adjusted herd immunity. This relationship is statistically significant at a $99 \%$ confidence interval using a holdout sample to assess fit to model (actual reported deaths vs modeled herd immunity adjusted deaths). Importantly, this analysis is relative to the historic level of social restrictions and older adults continue to have high rates of susceptibility. This suggests that an easing of social restrictions absent vaccinations could lead to a second wave of infections, reported cases and deaths. 
Further, analysis of actual and projected vaccine administrations-considering volume, age distribution, efficacy by dose and lag time -are quantitatively demonstrated to have had nominal impact on death trends prior to mid-February and therefore cannot explain the decline in deaths which began in late December. However, vaccination remains a critical tool to protect individuals without naturally derived immunity, particularly older adults who have a much higher morbidity and mortality rate and very low cumulative rate of naturally derived immunity.

\section{Implications and Recommendations}

Using deaths and IFR to estimate incidence provides an important epidemiology tool as age specific deaths are available weekly, by state and by key demographic characteristics. Clinical analysis can quantitatively estimate differences in IFR across these characteristic and allow for more granular understanding of disease spread, relative risk analysis and projected death trends at a national and state level. These analyses can be used to inform public policy, individual health choices and vaccination policies particularly in a vaccine supply constrained environment. The ability to quantify and predict the precipitous decline in deaths provides a high degree of validation of the model as well as the relational sensitivity of underlying assumptions related to age specific IFR and durability of immunity.

\section{Caveats and Limitations}

This analysis is not meant to determine the herd immunity threshold, IFR or cumulative immunity levels, but rather quantitatively describes the relational sensitivity between these variables and establishes boundaries when viewed in the context of known variables (eg. IFR or cumulative deaths). This model could benefit from further sensitivity analysis and monte carlo simulations to pressure test the relational sensitivity of key assumptions.

Predictive trends quantified in this analysis are relevant in the context of current/historical social distancing and mask wearing behaviors. As of this writing, older adults still have very low levels of naturally acquired immunity and vaccination and are therefore still "at high risk" of infection, morbidity, and mortality even in the context of herd immunity. Changes in social distancing policy and behaviors could shift herd immunity trend dynamics negatively.

Herd immunity analysis is also relevant in the context of current/historic COVID-19 strains in circulation. Future relative risk may be different if emerging genetic variants prove to have higher infectiousness and/or morbidity than historic variants. As of this publication new strains are believed to be more infectious but there is no strong evidence to support higher morbidity.

Age specific relative risks contextualized in this analysis are meant to represent population averages. Individuals may have higher or lower actual risk based on other risk factors. Additionally, herd immunity 
trends will not be able to drive infections down to the point of disease elimination. As such, individual risk may be significantly reduced due to herd immunity but not eliminated.

A relatively short period of time was used to project age specific death trends. Additionally, the number of deaths among individuals less than 15 years old were too thin to reliably trend and estimates of incidence for this age group are the least reliable for that reason. However, incidence among children should have nominal impact on predicted reported death trends.

This analysis does not consider the impact of changes in social distancing (around the holidays), weather patterns, political and social media influences on behavior as the underlying dynamics of epidemiology (IFR and herd immunity) can account for a significant portion of the variability in reported death trends.

\section{References}

1. Centers for Disease Control and Prevention. COVID Data Tracker. Trends in Number of COVID-19 Cases and Deaths in the US Reported to CDC, by State/Territory. [Online] February 13, 2021. https://covid.cdc.gov/covid-data-tracker/\#trends_dailytrendscases .

2. Statista Research Department. Projected path to reach estimated COVID-19 herd immunity at $70 \%$ in the United States as of February 21, 2021*. statista. [Online] February 26, 2021. https://www.statista.com/statistics/1198638/share-population-covid-immunity-us-by-type/.

3. Donald G. McNeil Jr. How Much Herd Immunity Is Enough? The New York Times. February 21, 2021.

4. On the Effect of Age on the Transmission of SARS-CoV-2 in Households, Schools, and the Community. Edward Goldstein, et al. 3, October 2020, The Journal of Infectious Diseases, Vol. 223, pp. 362-369.

5. A mathematical model reveals the influence of population heterogeneity on herd immunity to SARSCoV-2. Tom Britton, et al. 6505, August 2020, Science, Vol. 369, pp. 846-849.

6. Centers for Disease Control and Prevention. COVID-19. COVID-19 Pandemic Planning Scenarios. [Online] September 10, 2020. https://www.cdc.gov/coronavirus/2019-ncov/hcp/planningscenarios.html.

7. Estimation of SARS-COV-2 mortality during the early stages of an epidemic: A modeling study in Hubei, China, and six regions in Europe. Hauser A, Counotte MJ, Margossian CC, Konstantinoudis G, Low N, Althaus CL, et al. 2020, PLoS Med, Vol. 17(7): e1003189.

8. Assessing the age specificity of infection fatality rates for COVID-19: systematic review, metaanalysis, and public policy implications. Andrew T Levin, et al. December 2020, European Journal of Epidemiology, Vol. 35, pp. 1123-1138.

9. Douglas L. Rothman, Jessica E. Rothman, and Gerard Bossard. Is Covid-19 growing less lethal? The infection fatality rate says "no'. August 24, 2020.

10. Trends in COVID-19 Risk-Adjusted Mortality Rates. Leora I Horwitz, MD, MHS. October 23, 2020, Journal of Hospital Medicine. 
11. A systematic review and meta-analysis of published research data on COVID-19 infection-fatality rates. Gideon Meyerowitz-Katz, Lea Merone. July 7, 2020, medRxiv.

12. Centers for Disease Control and Prevention. COVID-19. SARS-CoV-2 Variants. [Online] January 21, 2021. https://www.cdc.gov/coronavirus/2019-ncov/cases-updates/variant-surveillance/variantinfo.html.

13. Immunological memory to SARS-CoV-2 assessed for up to 8. Dan, J. M. 2021, Science, p. 10.1126/science.abf4063 (2021).

14. Functional SARS-CoV-2-sperific immune memory persists after mild COVID-19. Rodda, Lauren B. August 13, 2020, PMC, pp. doi: 10.21203/rs.3.rs-57112/v1.

15. ModernaTX, Inc. Vaccines and Related Biological Products Advisory Committee Meeting, FDA Briefing Document. 2020. p. 28.

16. United States Census Bureau. Age and Sex Composition in the United States: 2019. Detailed Tables by Age and Sex. [Online] 2019. https://www.census.gov/data/tables/2019/demo/age-and-sex/2019-agesex-composition.html.

17. Centers for Disease Control and Prevention. COVID Data Tracker. COVID-19 Vaccinations in the United States. [Online] February 24, 2021. https://covid.cdc.gov/covid-data-tracker/\#vaccinations.

18. Murphy, JOe. Biden pledged 150 million Covid vaccinations in 100 days. This is what the numbers say. NBC News. January 26, 2021, pp. https://www.nbcnews.com/politics/white-house/150-millionvaccinations-tracker-biden-goal-n1255716.

19. Prevention, Centers for Disease Control and. National Center for Health Statistics. Weekly Updates by Select Demographic and Geographic Characteristics. [Online] February 13, 2021. https://www.cdc.gov/nchs/nvss/vsrr/covid_weekly/index.htm\#AgeAndSex.

\section{Additional Information}

I have no disclosures, funding or affiliations to declare.

Supplemental Information is available for this paper.

The datasets and code generated during and/or analyzed during this study are available [TBD].

\section{Figures}




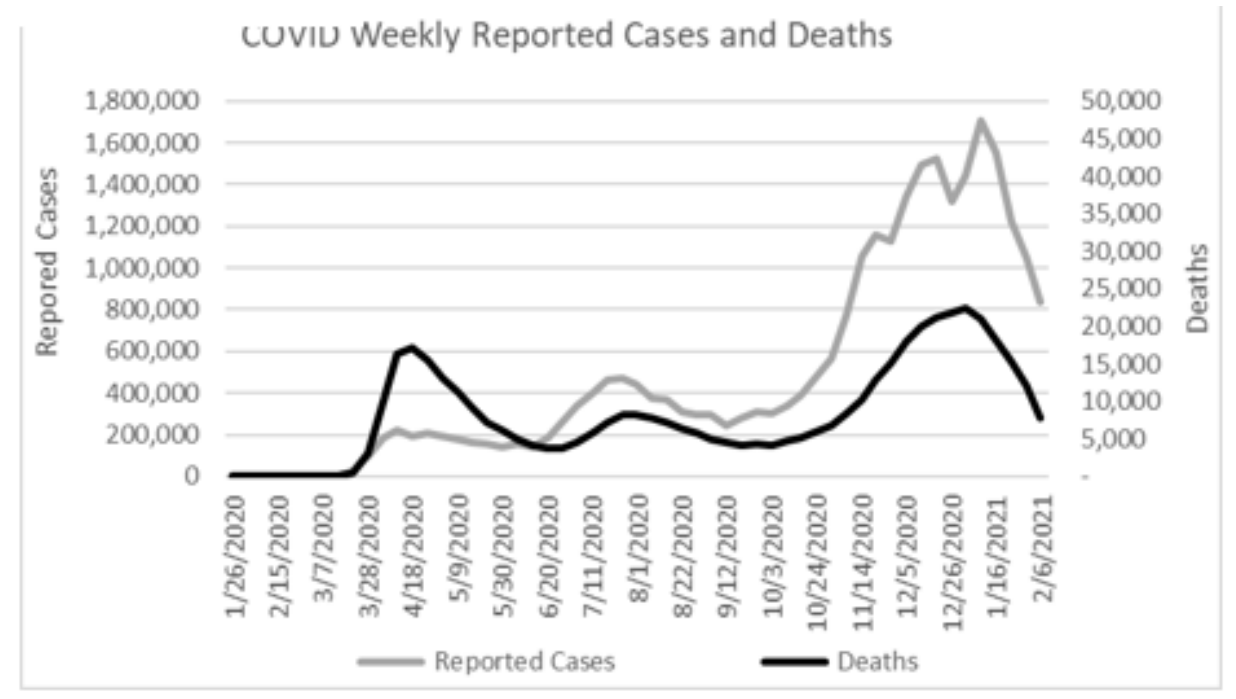

Figure 1

COVID Weekly Reported Cases and Death

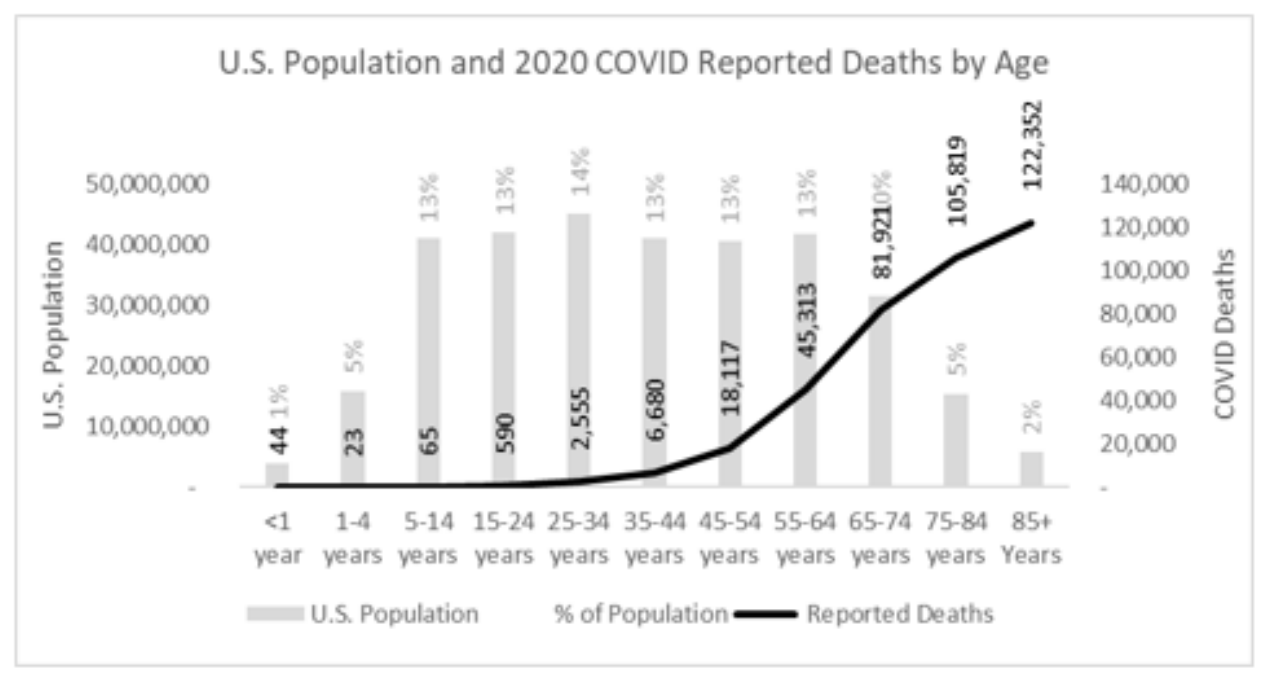

Figure 2

U.S. Population and 2020 COVID Reported Deaths by Age 


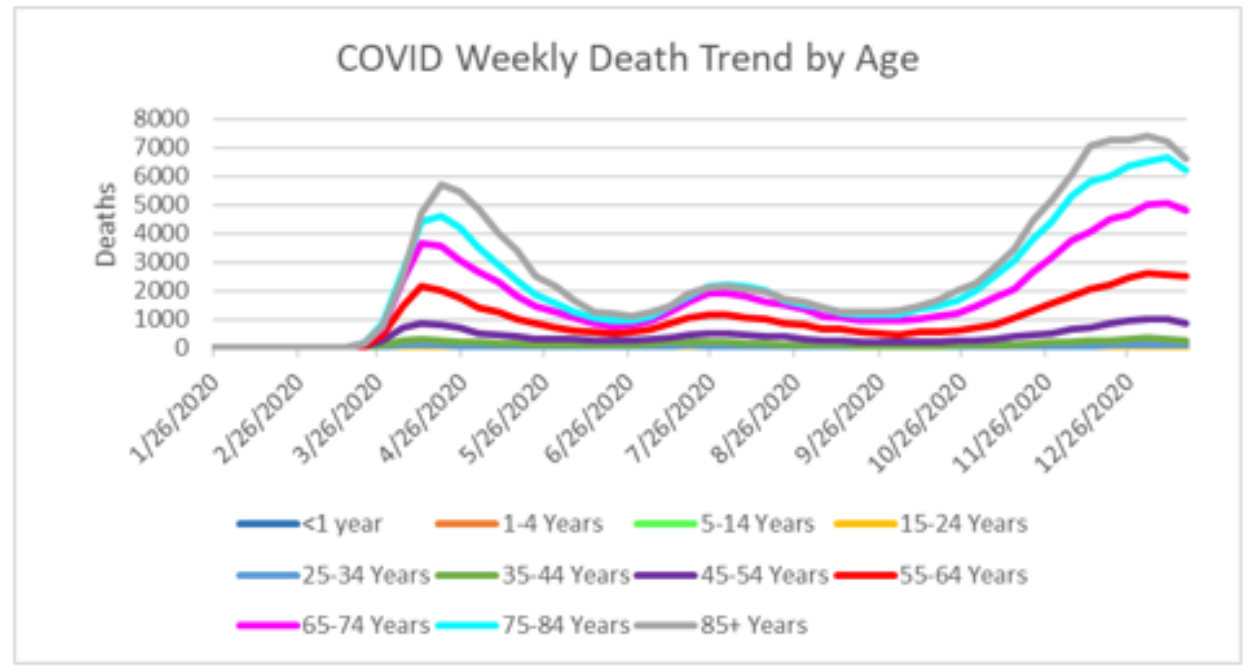

Figure 3

COVID Weekly Death Trend by Age

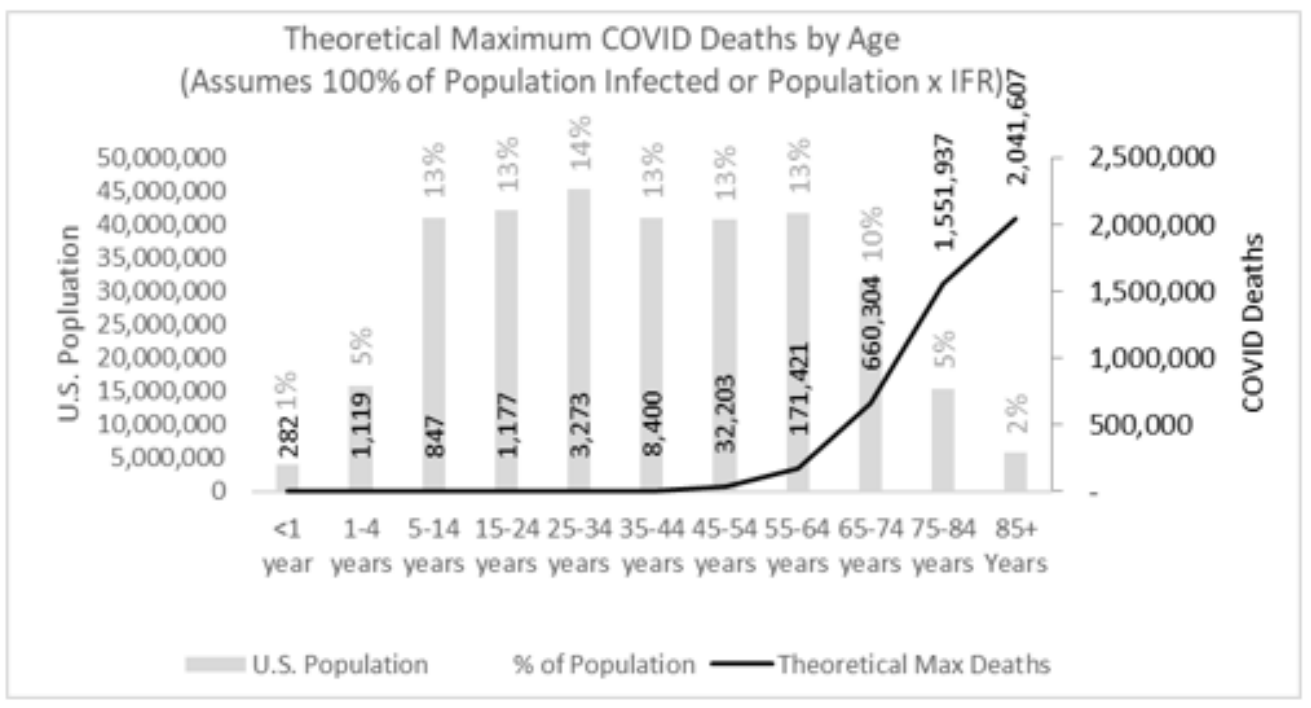

Figure 4

Theoretical Maximum Reported Deaths by Age

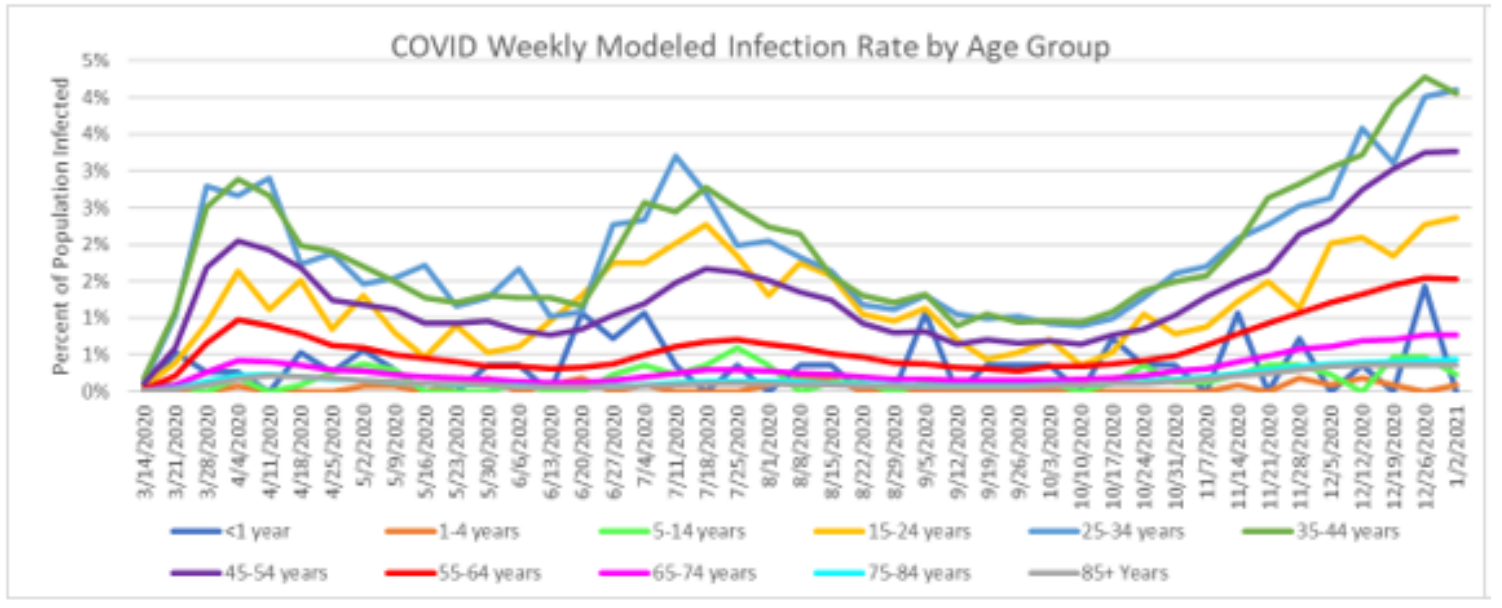


Figure 5

COVID Weekly Modeled Infection Rate by Age Group

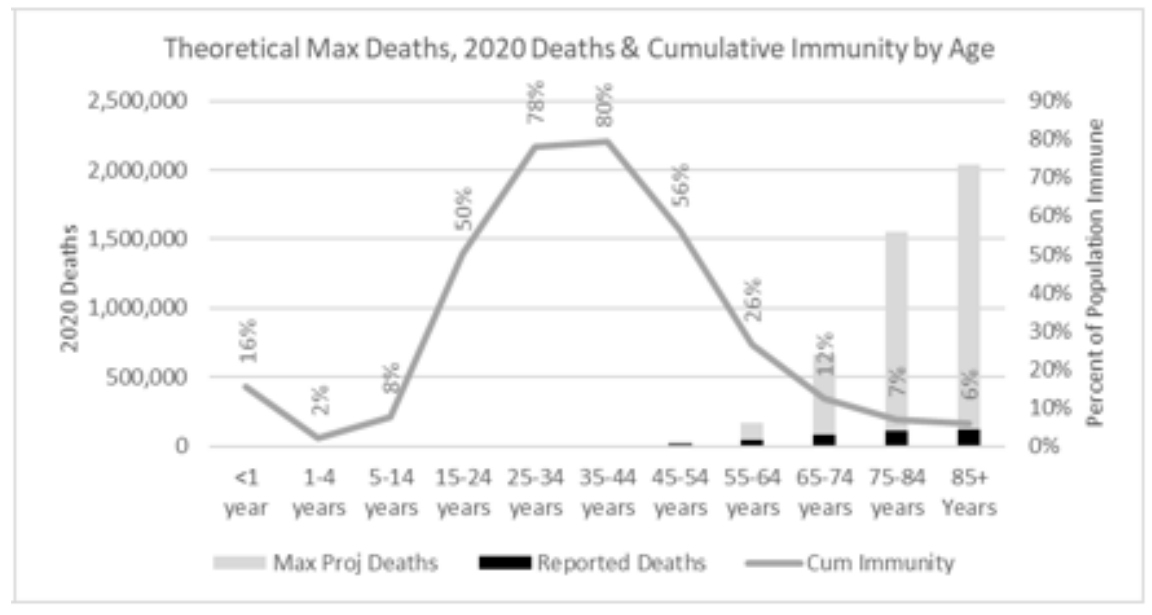

Figure 6

Theoretical Max Deaths, 2020 Deaths \& Cumulative Immunity by Age

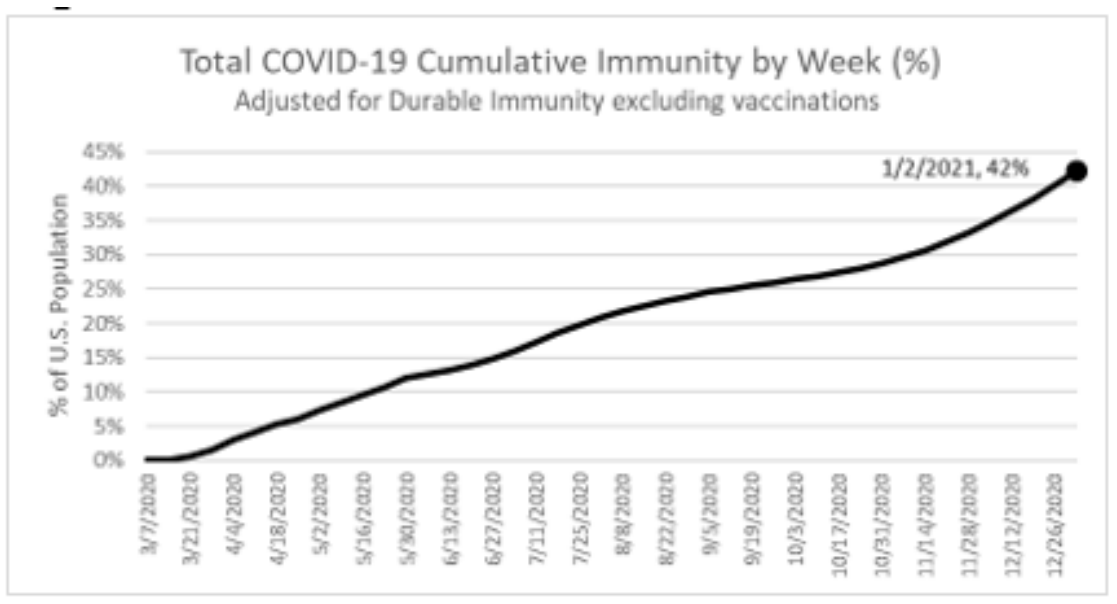

\section{Figure 7}

Total COVID-19 Cumulative Immunity by Week

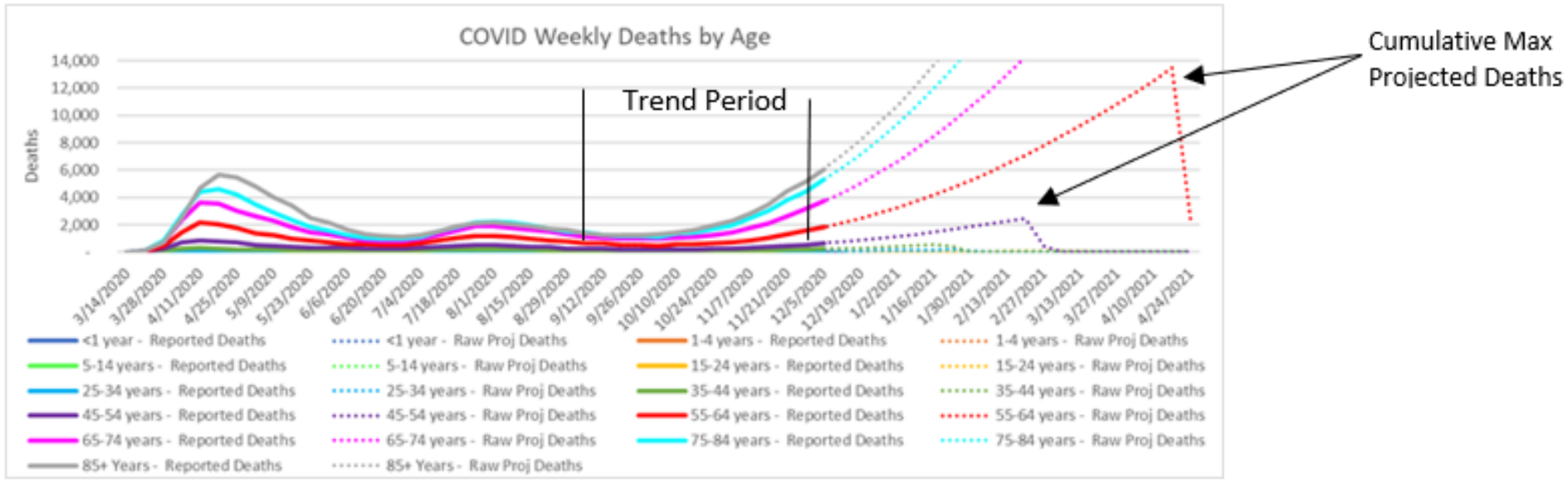


Figure 8

COVID Weekly Deaths by Age

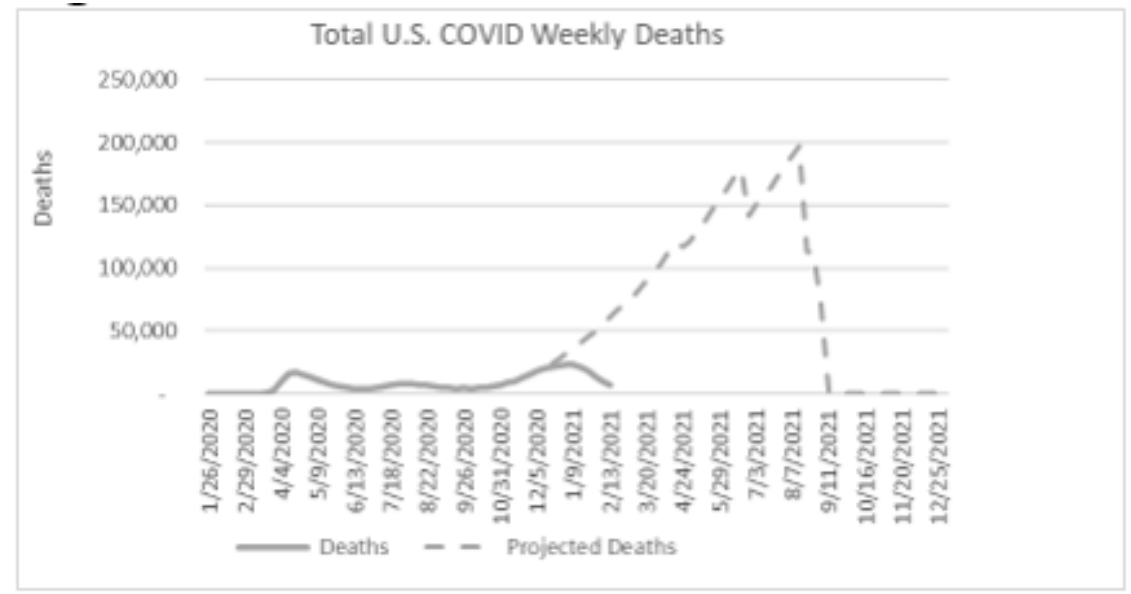

\section{Figure 9}

\section{Total U.S. COVID Weekly Deaths}

Total U.S. COVID Weekly Infections and Reported Cases

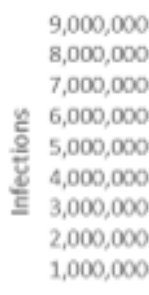

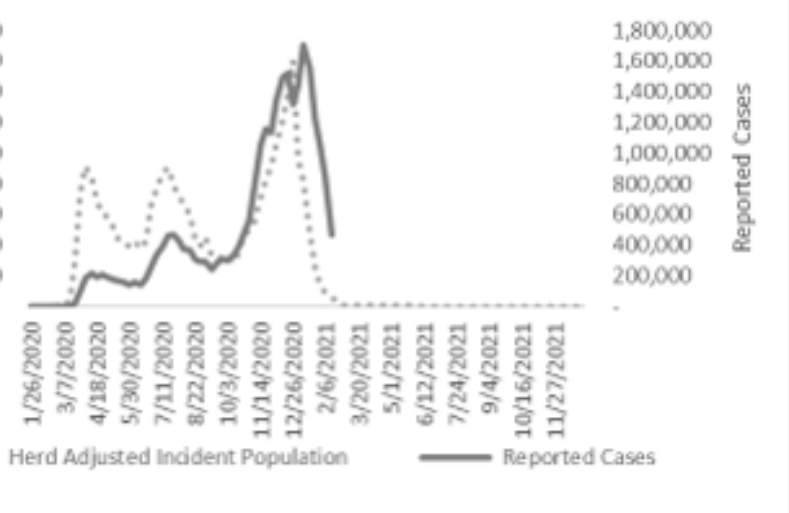

Figure 10

Total U.S. COVID Weekly Infections and Reported Cases

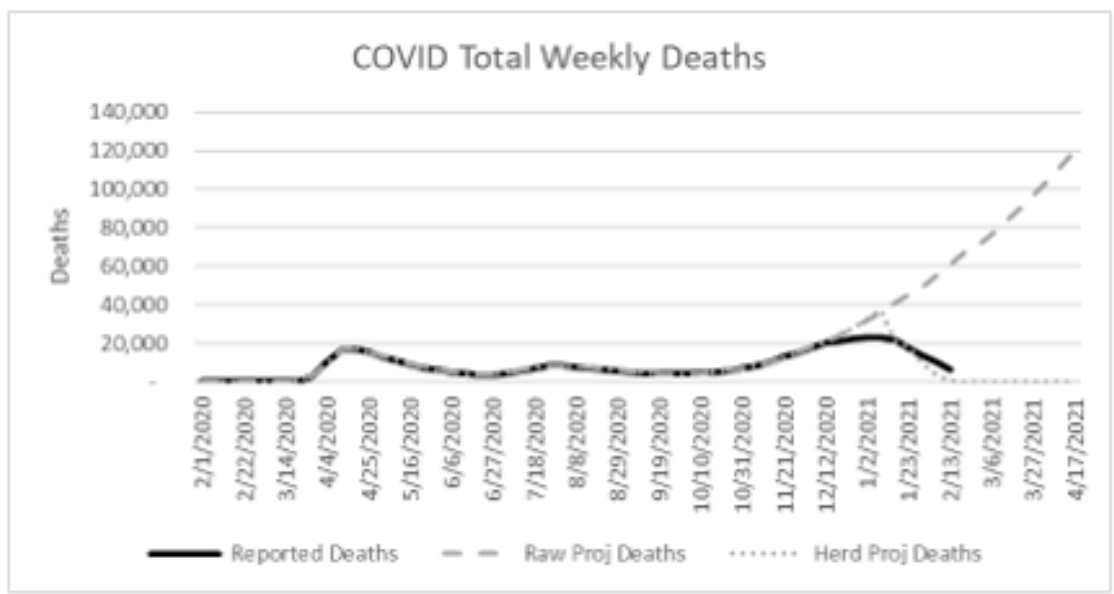


Figure 11

COVID Total Weekly Deaths

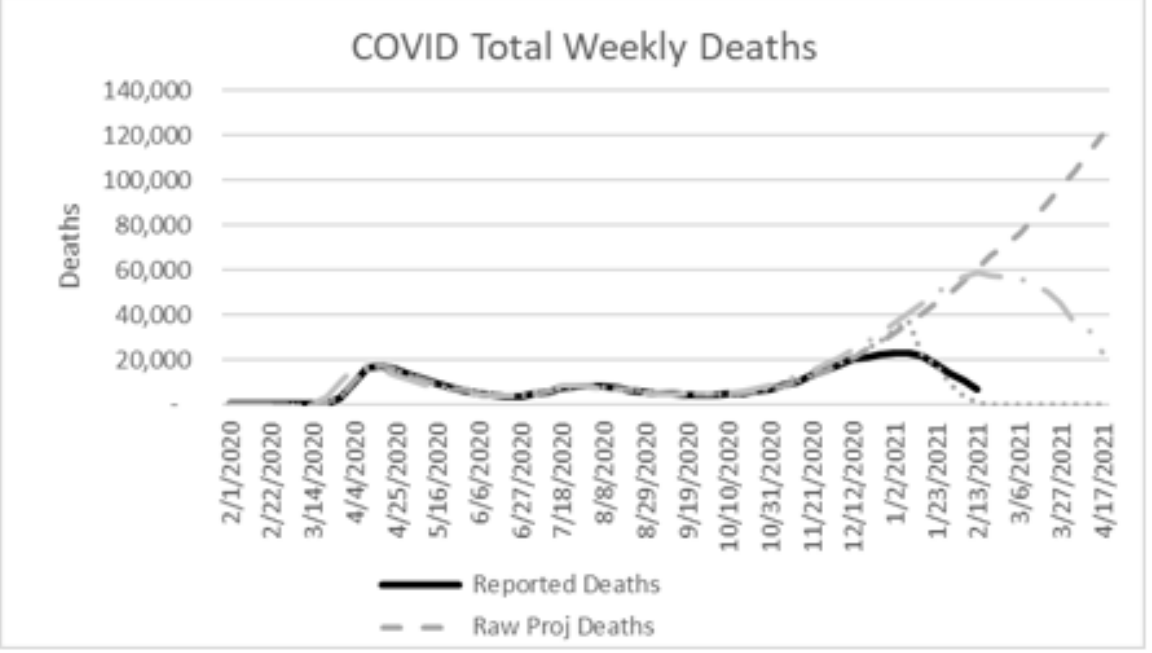

\section{Figure 12}

\section{COVID Total Weekly Deaths}

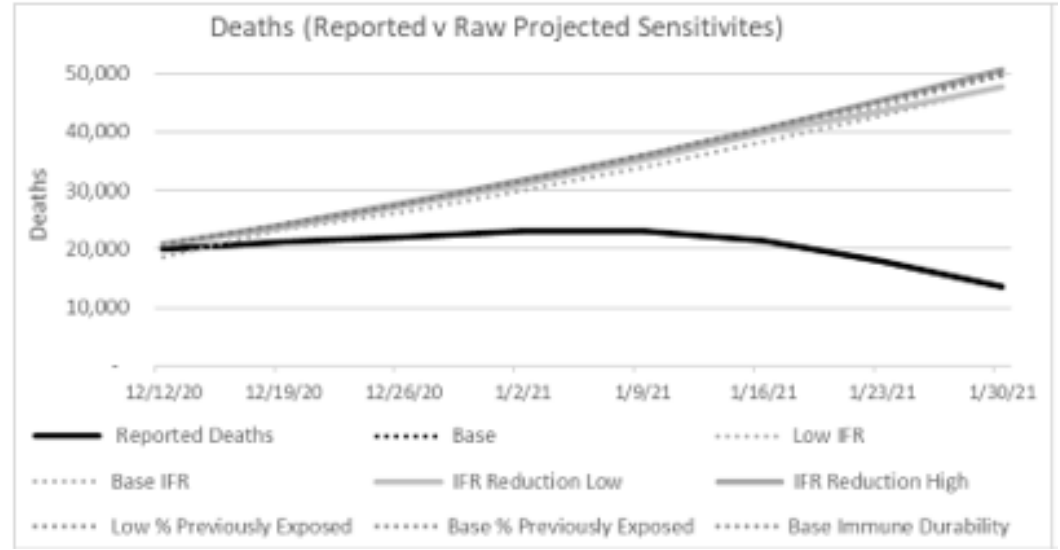

Figure 13

Deaths (Reported v Raw Projected Sensitivities)

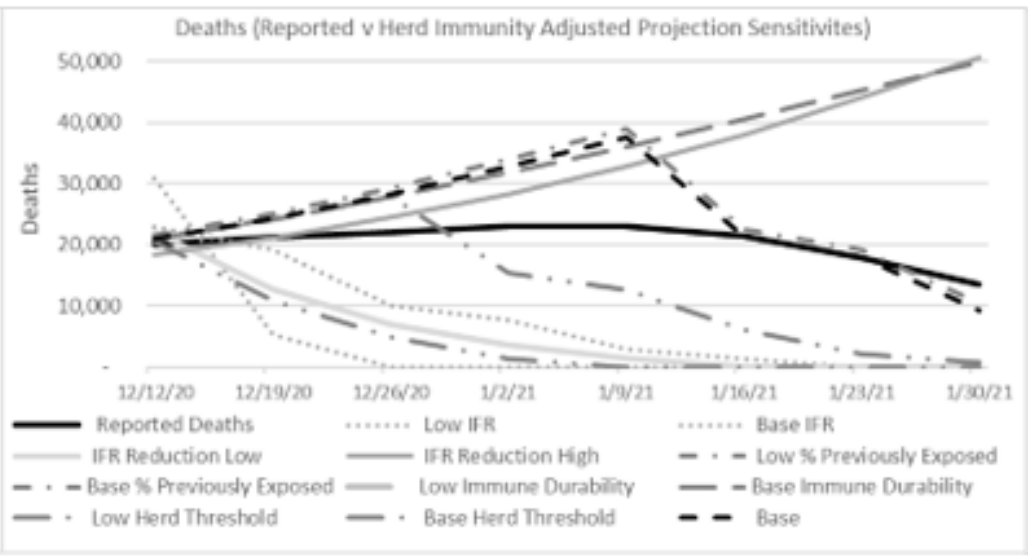

Figure 14 
Deaths (Reported v Herd Immunity Adjusted Projection Sensitivities)

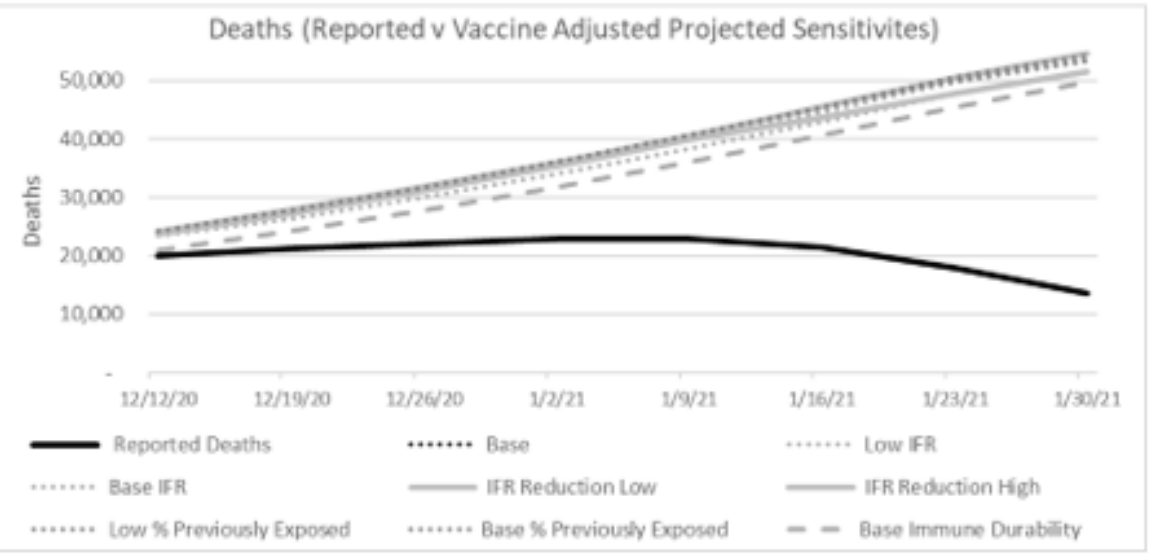

Figure 15

Deaths (Reported v Vaccine Adjusted Projected Sensitivities)

\section{Supplementary Files}

This is a list of supplementary files associated with this preprint. Click to download.

- COVIDEpidemiologyandHerdImmunitySCIENTIFICREPORTSSUPPLIMENTALMETHODS.docx

- SupplementalFigure.docx 